\title{
DYNAMIC BEHAVIOUR OF THE SOLUTIONS ON A CLASS OF COUPLED VAN DER POL EQUATIONS WITH DELAYS
}

\section{CHUNHUA FENG}

Department of Mathematics and Computer Science

Alabama State University

Montgomery, AL, 36104

USA

e-mail: cfeng@alasu.edu

\begin{abstract}
In this paper, a class of coupled van der Pol equations with time delays is investigated. By means of mathematical analysis approach, some sufficient conditions to guarantee the existence of oscillatory solutions for the model are obtained. Computer simulations are provided to demonstrate the proposed results.
\end{abstract}

\section{Introduction}

For a class of coupled van der Pol equations of the form:

$$
\left\{\begin{array}{c}
u_{1}^{\prime \prime}(t)+\varepsilon_{1}\left(u_{1}^{2}(t)-a_{1}\right) u_{1}^{\prime}(t)+c_{11} u_{1}(t)+c_{12} u_{2}(t)+c_{13} u_{3}(t)+\cdots+c_{1 n} u_{n}(t)=0 \\
u_{2}^{\prime \prime}(t)+\varepsilon_{2}\left(u_{2}^{2}(t)-a_{2}\right) u_{2}^{\prime}(t)+c_{21} u_{1}(t)+c_{22} u_{2}(t)+c_{23} u_{3}(t)+\cdots+c_{2 n} u_{n}(t)=0 \\
\cdots \\
u_{n}^{\prime \prime}(t)+\varepsilon_{n}\left(u_{n}^{2}(t)-a_{n}\right) u_{n}^{\prime}(t)+c_{n 1} u_{1}(t)+c_{n 2} u_{2}(t)+c_{n 3} u_{3}(t)+\cdots+c_{n n} u_{n}(t)=0
\end{array}\right.
$$

2010 Mathematics Subject Classification: 34K11.

Keywords and phrases: coupled van der Pol equation, delay, oscillation.

Received November 16, 2016

(C) 2016 Scientific Advances Publishers 
where $n \geq 1, \varepsilon_{i}>0, c_{i j} \in R, 1 \leq i, j \leq n$. Many good results have appeared (see (1)-(9)). For the case $n=2$, Hirano and Rybicki [1] have investigated the existence of limit cycles by using $S^{1}$ - degree theory, and their argument remains valid for the case that $n>2$. Rompala et al. [8] considered a system of three van der Pol oscillators, which coupled as follows:

$$
\left\{\begin{array}{l}
x^{\prime \prime}(t)-\varepsilon\left(1-x^{2}(t)\right) x^{\prime}(t)+x(t)=\varepsilon \mu[w(t)-x(t)] \\
y^{\prime \prime}(t)-\varepsilon\left(1-y^{2}(t)\right) y^{\prime}(t)+y(t)=\varepsilon \mu[w(t)-y(t)] \\
w^{\prime \prime}(t)-\varepsilon\left(1-w^{2}(t)\right) w^{\prime}(t)+p^{2} w(t)=\varepsilon \mu[x(t)-w(t)]+\varepsilon \mu[w(t)-y(t)] .
\end{array}\right.
$$

For a ring of four mutually coupled biological systems described by coupled van der Pol oscillators:

$$
\left\{\begin{array}{l}
x_{1}^{\prime \prime}(t)-\varepsilon\left(1-x_{1}^{2}(t)\right) x_{1}^{\prime}(t)+x_{1}(t)=k_{1}\left(x_{2}(t)-x_{1}(t)\right)+k_{2}\left(x_{4}(t)-x_{1}(t)\right), \\
x_{2}^{\prime \prime}(t)-\varepsilon\left(1-x_{2}^{2}(t)\right) x_{2}^{\prime}(t)+x_{2}(t)=k_{1}\left(x_{1}(t)-x_{2}(t)\right)+k_{2}\left(x_{3}(t)-x_{2}(t)\right), \\
x_{3}^{\prime \prime}(t)-\varepsilon\left(1-x_{3}^{2}(t)\right) x_{3}^{\prime}(t)+x_{3}(t)=k_{1}\left(x_{4}(t)-x_{3}(t)\right)+k_{2}\left(x_{2}(t)-x_{3}(t)\right), \\
x_{4}^{\prime \prime}(t)-\varepsilon\left(1-x_{4}^{2}(t)\right) x_{4}^{\prime}(t)+x_{4}(t)=k_{1}\left(x_{3}(t)-x_{4}(t)\right)+k_{2}\left(x_{1}(t)-x_{4}(t)\right) .
\end{array}\right.
$$

The stability boundaries and the main dynamical states have been considered on the stability maps in the $\left(k_{1}, k_{2}\right)$ plane [9].

It is well known that the time delay is inevitable in many physical and biological phenomena, such as manufacturing process, nuclear reactors, rocket motors, mechanical controlling systems, population dynamics, and so on. Naturally the time delay coupled van der Pol equations also have been extensively studied by many researchers [10-23]. For example, Wirkus et al. [10] have considered the dynamics of two weakly coupled van der Pol oscillators, in which the coupling terms have time delays: 


$$
\left\{\begin{array}{l}
x_{1}^{\prime \prime}(t)+\varepsilon\left(x_{1}^{2}(t)-1\right) x_{1}^{\prime}(t)+x_{1}(t)=\varepsilon \alpha x_{2}^{\prime}\left(t-\tau_{2}\right), \\
x_{2}^{\prime \prime}(t)+\varepsilon\left(x_{2}^{2}(t)-1\right) x_{2}^{\prime}(t)+x_{2}(t)=\varepsilon \alpha x_{1}^{\prime}\left(t-\tau_{1}\right) .
\end{array}\right.
$$

Li et al. [11] have studied the coupled van der Pol oscillators with two kinds of delays:

$$
\left\{\begin{array}{l}
y_{1}^{\prime \prime}(t)+w_{1}^{2} y_{1}(t)-\varepsilon\left(1-y_{1}^{2}(t)\right) y_{1}^{\prime}(t)=\varepsilon \alpha\left[y_{2}(t-\tau)+y_{2}^{\prime}(t-\tau)\right], \\
y_{2}^{\prime \prime}(t)+w_{2}^{2} y_{2}(t)-\varepsilon\left(1-y_{2}^{2}(t)\right) y_{2}^{\prime}(t)=\varepsilon \alpha\left[y_{1}(t-\tau)+y_{1}^{\prime}(t-\tau)\right] .
\end{array}\right.
$$

Zhang and $\mathrm{Gu}$ [12] used the theory of normal form and central manifold theorem to discuss the following time delay system:

$$
\left\{\begin{array}{l}
x_{1}^{\prime \prime}(t)+\varepsilon\left(x_{1}^{2}(t)-1\right) x_{1}^{\prime}(t)+x_{1}(t)=\alpha\left[y_{1}\left(t-\tau_{2}\right)-x_{1}(t)\right], \\
y_{1}^{\prime \prime}(t)+\varepsilon\left(y_{1}^{2}(t)-1\right) y_{1}^{\prime}(t)+x_{1}(t)=\alpha\left[x_{1}\left(t-\tau_{1}\right)-y_{1}(t)\right] .
\end{array}\right.
$$

Motivated by the above models, in this paper, we consider the following time delays model:

$$
\left\{\begin{array}{c}
u_{1}^{\prime \prime}(t)+\varepsilon_{1}\left(u_{1}^{2}(t)-a_{1}\right) u_{1}^{\prime}(t)+c_{11} u_{1}\left(t-r_{1}\right)+c_{12} u_{2}\left(t-r_{2}\right)+\cdots+c_{1 n} u_{n}\left(t-r_{n}\right)=0 \\
u_{2}^{\prime \prime}(t)+\varepsilon_{2}\left(u_{2}^{2}(t)-a_{2}\right) u_{2}^{\prime}(t)+c_{21} u_{1}\left(t-r_{1}\right)+c_{22} u_{2}\left(t-r_{2}\right)+\cdots+c_{2 n} u_{n}\left(t-r_{n}\right)=0 \\
\cdots \\
u_{n}^{\prime \prime}(t)+\varepsilon_{n}\left(u_{n}^{2}(t)-a_{n}\right) u_{n}^{\prime}(t)+c_{n 1} u_{1}\left(t-r_{1}\right)+c_{n 2} u_{2}\left(t-r_{2}\right)+\cdots+c_{n n} u_{n}\left(t-r_{n}\right)=0
\end{array}\right.
$$

where $0<a_{i}$ and $0<\varepsilon_{i} \ll 1$ for each $i=1,2, \ldots, n . c_{i j} \in R(1 \leq i, j \leq n)$, $r_{i}(1 \leq i \leq n)$ are time delays. Our aim is to investigate the dynamic behaviour of $n$ coupled oscillators.

\section{Preliminaries}

For convenience, letting $u_{i}(t)=x_{2 i-1}(t), u_{1}^{\prime}(t)=x_{2 i}(t), r_{i}=\tau_{2 i-1}$, $c_{i j}=b_{2 i, 2 j-1}(1 \leq i, j \leq n)$, then the coupled system (7) can be written as the following: 


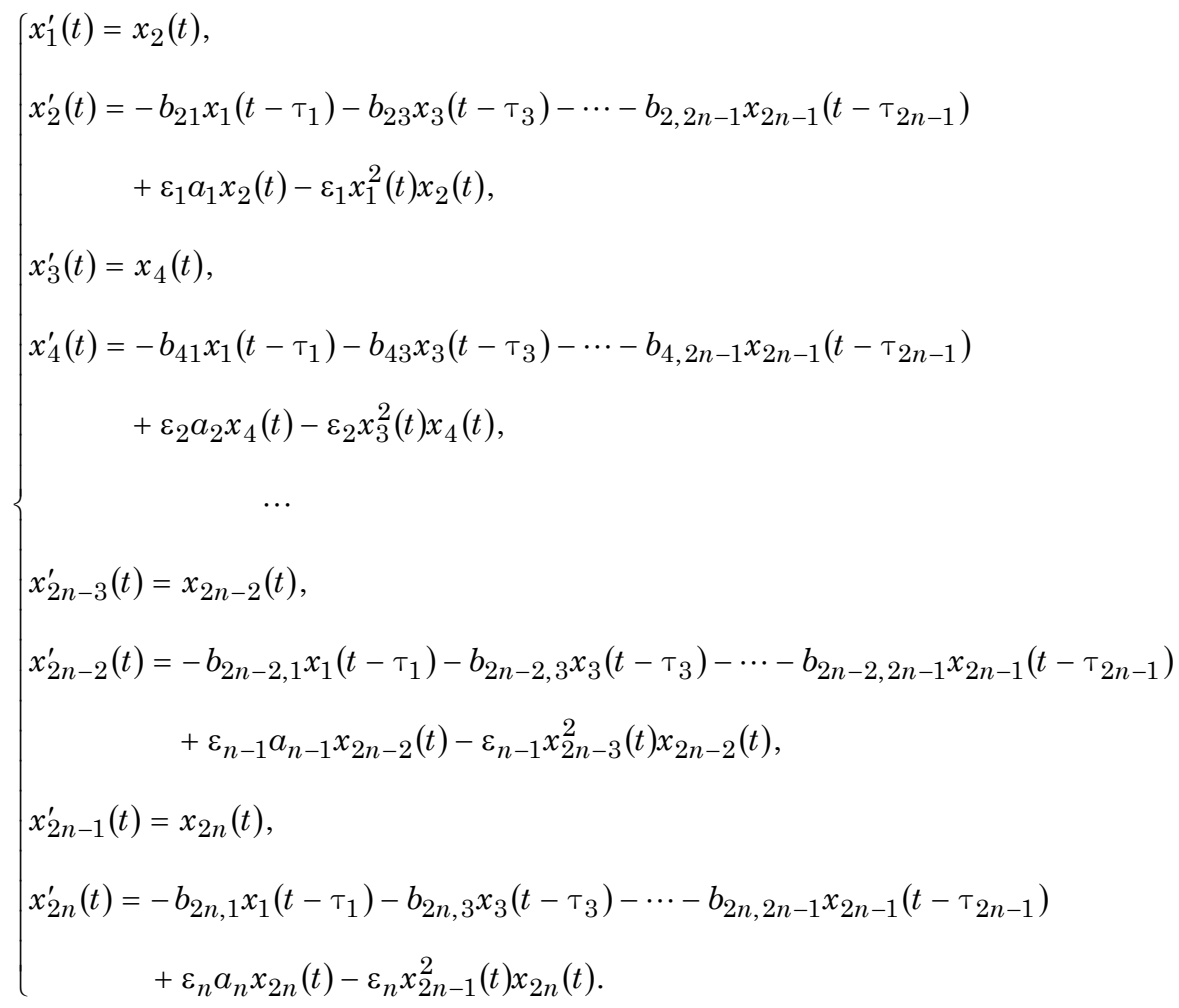

Obviously, the origin $x_{k}=0(k=1,2, \ldots, 2 n)$ is an equilibrium of system (8). The linearization of the coupled system (8) at origin is 


$$
\left\{\begin{array}{l}
x_{1}^{\prime}(t)=x_{2}(t), \\
x_{2}^{\prime}(t)=-\sum_{k=1}^{n} b_{2,2 k-1} x_{2 k-1}\left(t-\tau_{2 k-1}\right)+\varepsilon_{1} a_{1} x_{2}(t), \\
x_{3}^{\prime}(t)=x_{4}(t), \\
x_{4}^{\prime}(t)=-\sum_{k=1}^{n} b_{4,2 k-1} x_{2 k-1}\left(t-\tau_{2 k-1}\right)+\varepsilon_{2} a_{2} x_{4}(t), \\
\quad \ldots \\
x_{2 n-3}^{\prime}(t)=x_{2 n-2}(t), \\
x_{2 n-2}^{\prime}(t)=-\sum_{k=1}^{n} b_{2 n-2,2 k-1} x_{2 k-1}\left(t-\tau_{2 k-1}\right)+\varepsilon_{n-1} a_{n-1} x_{2 n-2}(t), \\
x_{2 n-1}^{\prime}(t)=x_{2 n}(t), \\
x_{2 n}^{\prime}(t)=-\sum_{k=1}^{n} b_{2 n, 2 k-1} x_{2 k-1}\left(t-\tau_{2 k-1}\right)+\varepsilon_{n} a_{n} x_{2 n}(t) .
\end{array}\right.
$$

The system (9) can be expressed in the following matrix form:

$$
X^{\prime}(t)=A X(t)+B X(t-\tau),
$$

where $X(t)=\left[x_{1}(t), x_{2}(t), \cdots, x_{2 n}(t)\right]^{T}, X(t-\tau)=\left[x_{1}\left(t-\tau_{1}\right), 0, x_{3}\left(t-\tau_{3}\right)\right.$, $\left.0, x_{5}\left(t-\tau_{5}\right), 0, \cdots, x_{2 n-1}\left(t-\tau_{2 n-1}\right), 0\right]^{T}$. Both $A=\left(a_{i j}\right)_{2 n \times 2 n} \quad$ and $B=\left(b_{i j}\right)_{2 n \times 2 n}$ are $2 n \times 2 n$ matrices as follows: 


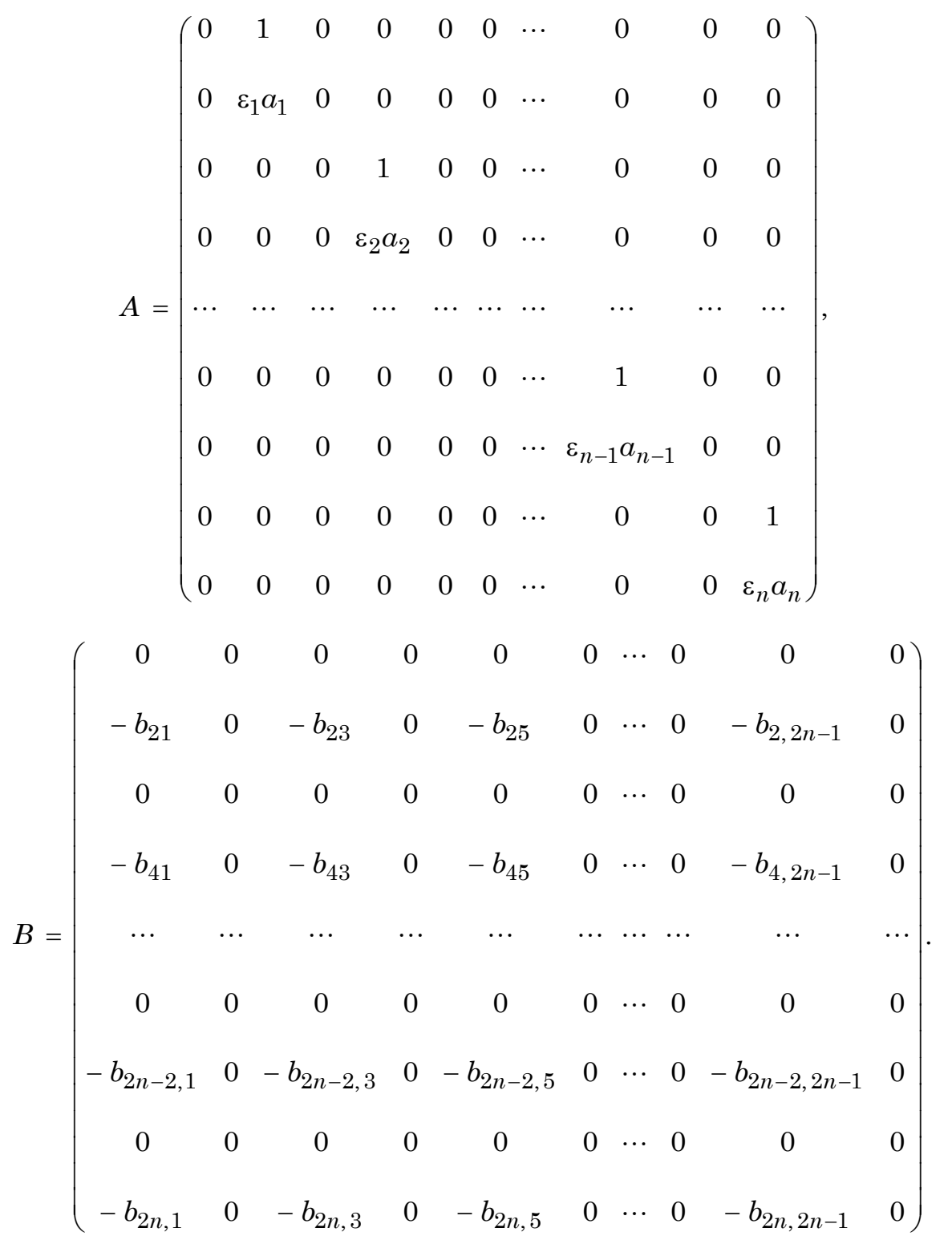

Lemma 1. Assume that the matrix $C(=A+B)$ is a nonsingular matrix, then system (9) has a unique equilibrium, implying that system (8) has a unique equilibrium. 
Proof. An equilibrium point $x^{*}=\left[x_{1}^{*}, x_{2}^{*}, \cdots, x_{2 n}^{*}\right]^{T}$ of system (9) is a constant solution of the following algebraic equation:

$$
A x^{*}+B x^{*}=(A+B) x^{*}=C x^{*}=0 .
$$

Since $C(=A+B)$ is a $2 n \times 2 n$ matrix, according to the linear algebraic knowledge, if $C$ is a singular matrix, Equation (11) may have infinitely many solutions. However, if $C$ is a nonsingular matrix, Equation (11) has only one solution, namely, the trivial solution. Implying that system (9) has a unique equilibrium. Noting that in system (8), the nonlinear terms $\varepsilon_{i} x_{2 i-1}^{2}(t) x_{2 i}(t)(i=1,2, \ldots, n)$ are higher order infinitesimal when $x_{2 i-1}$ and $x_{2 i}(i=1,2, \ldots, n)$ tend to zero. Therefore, system (9) has a unique equilibrium suggests that system (8) has a unique trivial solution.

Lemma 2. All solutions of system (8) are uniformly bounded.

Proof. To prove the boundedness of the solutions in system (8), we construct a Lyapunov function $V(t)=\sum_{i=1}^{2 n} \frac{1}{2} x_{i}^{2}(t)$. Calculating the derivative of $V(t)$ through system (8), one get

$$
\begin{aligned}
\left.V^{\prime}(t)\right|_{(8)}= & \sum_{i=1}^{2 n} x_{i}(t) x_{i}^{\prime}(t) \\
= & x_{1} x_{2}+x_{2}\left\{-\sum_{i=1}^{n} b_{2,2 i-1} x_{2 i-1}\left(t-\tau_{2 i-1}\right)\right\}+\varepsilon_{1} a_{1} x_{2}^{2}-\varepsilon_{1} x_{1}^{2} x_{2}^{2} \\
& +x_{3} x_{4}+x_{4}\left\{-\sum_{i=1}^{n} b_{4,2 i-1} x_{2 i-1}\left(t-\tau_{2 i-1}\right)\right\}+\varepsilon_{2} a_{2} x_{4}^{2}-\varepsilon_{2} x_{3}^{2} x_{4}^{2} \\
& +\cdots+x_{2 n-1} x_{2 n}+x_{n}\left\{-\sum_{i=1}^{2 n} b_{2 n, 2 i-1} x_{2 i-1}\left(t-\tau_{2 i-1}\right)\right\} \\
& +\varepsilon_{n} a_{n} x_{2 n}^{2}-\varepsilon_{n} x_{2 n-1}^{2} x_{2 n}^{2}
\end{aligned}
$$




$$
\begin{aligned}
\leq & -\sum_{i=1}^{n} \varepsilon_{i} x_{2 i-1}^{2} x_{2 i}^{2}+\sum_{i=1}^{n}\left|x_{2 i-1}\right|\left|x_{2 i}\right|+\sum_{j=1}^{n} \sum_{i=1}^{n}\left|b_{2 j, 2 i-1}\right|\left|x_{2 j}\right|\left|x_{2 i-1}\right| \\
& +\sum_{i=1}^{n} \varepsilon_{i} a_{i} x_{2 i}^{2}
\end{aligned}
$$

Noting that as $x_{2 i-1} x_{2 i}$ and $x_{2 j} x_{2 i-1}(i, j=1,2, \ldots, n)$ tend to infinity, $x_{2 i-1}^{2} x_{2 i}^{2}(i=1,2, \ldots, n)$ are higher order infinity than $x_{2 i-1} x_{2 i}$ and $x_{2 j} x_{2 i-1}$. Therefore, there exists suitably large $L>0$ such that $\left.V^{\prime}(t)\right|_{(8)}<0$ as $\left|x_{i}\right| \geq L(i=1,2, \ldots, 2 n)$ since $0<\varepsilon_{i}(i=1,2, \ldots, 2 n)$.

This means that all solutions of system (8) and hence system (7) are bounded.

\section{Main Results}

Theorem 1. Assume that linearized system (9) has a unique equilibrium point, for given parameters values of $a_{i}, c_{i j}\left(=b_{2 i, 2 j-1}\right)$, and $\varepsilon_{i}(1 \leq i, j \leq n)$.

Let $\mu(A)=\max _{1 \leq i \leq n}\left\{1+\varepsilon_{i} a_{i}\right\}$ and $\|B\|=\max _{1 \leq i \leq n} \quad \sum_{i=1}^{n}\left|-b_{2 i, 2 j-1}\right|$. If the following condition holds:

$$
(|| B \|) e \tau_{*} \exp \left(-\tau_{*}|\mu(A)|\right)>1,
$$

where $\tau_{*}=\min \left\{\tau_{1}, \tau_{3}, \ldots, \tau_{2 n-1}\right\}$. Then the unique equilibrium of system (9) is unstable. System (7) generates a limit cycle, namely, a periodic solution.

Proof. We shall show that the unique equilibrium of system (9) is unstable. Consider $\tau_{1}=\tau_{3}=\ldots=\tau_{2 n-1}=\tau_{*}$ in system (10) as follows:

$$
X^{\prime}(t)=A X(t)+B X\left(t-\tau_{*}\right) .
$$

Let $z(t)=\sum_{i=1}^{2 n}\left|x_{i}(t)\right|$. Then we have

$$
\frac{d z(t)}{d t} \leq \mu(A) z(t)+\|B\| z\left(t-\tau_{*}\right) .
$$


Specially, for equation

$$
\frac{d w(t)}{d t}=\mu(A) w(t)+\|B\| w\left(t-\tau_{*}\right) .
$$

If the unique equilibrium of system (16) is stable, then the characteristic equation associated with (16) given by

$$
\lambda=\mu(A)+\|B\| e^{-\lambda \tau_{*}},
$$

will have a real negative root say $\lambda_{0}$, and we have from (17)

$$
\left|\lambda_{0}\right| \geq\|B\| e^{\left|\lambda_{0}\right| \tau_{*}}-|\mu(A)|
$$

Using the formula $e^{x} \geq e x$ for $x>0$, one can get

$$
1 \geq \frac{\|B\| e^{\left|\lambda_{0}\right| \tau_{*}}}{|\mu(A)|+\left|\lambda_{0}\right|}=\frac{\|B\| \tau_{*} e^{-|\mu(A)| \tau_{*}} e^{\left(|\mu(A)|+\left|\lambda_{0}\right|\right) \tau_{*}}}{\left(|\mu(A)|+\left|\lambda_{0}\right|\right) \tau_{*}} \geq\left(|| B \| e \tau_{*}\right) e^{-\tau_{*}|\mu(A)|} .
$$

The last inequality contradicts Equation (13). Hence, our claim regarding the instability of equilibrium of system (16) is valid. According to the comparison theorem of differential equation, we have $u(t) \leq w(t)$. Since the trivial solution is unstable in system (16), this implies that the trivial solution of (15) (thus system (10)) is unstable. Noting that in a time delay system, as the value of delay increases the instability of the unique equilibrium still maintains [22, 23]. So for any values of time delays the unique equilibrium of system (10) (or system (9)) is unstable. Recall that in system (8), the nonlinear terms $\varepsilon_{i} x_{2 i-1}^{2}(t) x_{2 i}(t)(i=1,2, \ldots, n)$ are higher order infinitesimal when $x_{2 i-1}$ and $x_{2 i}(i=1,2, \ldots, n)$ tend to zero. So the uniqueness and instability of the equilibrium of system (9) implies that system (8) has a unique unstable equilibrium. This suggests that the unique equilibrium of system (8) (or equivalent system (7)) is unstable. Since all solutions of system (7) are bounded, thus system (7) generates a limit cycle based on [24]. 
Theorem 2. Assume that linearized system (9) has a unique equilibrium point, for given parameters values of $a_{i}, c_{i j}$, and $\varepsilon_{i}(1 \leq i, j \leq n)$. If there exists a suitably large positive number $L$ such that the following inequality holds:

$$
L-\mu(A)-\|B\| e^{-L \tau_{*}}>0,
$$

Then the unique equilibrium of system (15) is unstable, implying that the unique equilibrium of system (9) is also unstable. System (7) generates a limit cycle, namely, a periodic solution.

Proof. We still consider system (16) and will prove that the trivial solution of system (16) is unstable. It is sufficient to show that the characteristic equation associated with (16) given by (17) has a real positive root. Noting that Equation (17) is a transcendental equation, the characteristic values may be complex numbers. However, we claim that Equation (17) has a real positive root. Let

$$
f(\lambda)=\lambda-\mu(A)-\|B\| e^{-\lambda \tau_{*}} .
$$

Obviously, $f(\lambda)$ is a continuous function of $\lambda$. Noting that $\tau_{*}>0$, $\mu(A)>0,\|B\|$ is bounded, and $f(0)=-\mu(A)-\|B\|<0$. On the other

hand, $f(L)=L-\mu(A)-\|B\| e^{-L \tau_{*}}>0$. According to the intermediate value theorem of continuous function, there exists a positive number $M$ such that $f(M)=0(M \in(0, L))$. In the other words, there exists a positive characteristic root of characteristic Equation (17). Therefore, the trivial solution of system (16) is unstable. Similar to Theorem 1, one can prove that system (8) generates a limit cycle, namely, a periodic solution.

\section{Simulation Results}

First we consider a three coupled van der Pol equation and used the form of system (8) for simulation. We fixed $\varepsilon_{1}=0.0054, \varepsilon_{2}=0.0065$, $\varepsilon_{3}=0.0068, a_{1}=1.2, a_{2}=1.25, a_{3}=1.3, b_{21}=0.6, b_{23}=0.2, b_{25}=0.5$, $b_{41}=0.2, b_{43}=0.15, b_{45}=0.12, b_{61}=0.4, b_{63}=0.3, b_{65}=0.25$. Therefore 
$\mu(A)=1.0068$ and $\|B\|=1.2$. We selected $\tau_{21}=0.4, \tau_{23}=4, \tau_{25}=3$, thus $\tau_{*}=0.4$ and $(\|B\|) e \tau_{*} \exp \left(-\tau_{*}|\mu(A)|\right)=1.1450>1$. Based on Theorem 1, this system generates a periodic solution (see Figure 1). When we increase the time delays as $\tau_{21}=5, \tau_{23}=6, \tau_{25}=8$, the dynamical behaviour still maintains (Figure 2). Then we discuss a four coupled van der Pol equation in system (8). We fixed $\varepsilon_{1}=0.0045$, $\varepsilon_{2}=0.0075, \varepsilon_{3}=0.005, \varepsilon_{4}=0.0045, a_{1}=1.15, a_{2}=1.25, a_{3}=1.24, a_{4}=1.32$, $b_{21}=0.95, b_{23}=1.5, b_{25}=0.68, b_{27}=1.02, b_{41}=1.15, b_{43}=4.85$, $b_{45}=1.48, b_{47}=1.12, b_{61}=0.05, b_{63}=0.025, b_{65}=1.06, b_{67}=0.52, b_{81}=0.15$, $b_{83}=0.25, b_{85}=0.08$, and $b_{87}=0.12$. Thus $\mu(A)=1.094,\|B\|=4.365$, time delays are $1.5,3.5,1.5,1.8$. So $\tau_{*}=1.5$. It is easy to check there exists $L>0$ such that condition (20) is satisfied. Based on Theorem 2, there exists a periodic solution of this system (see Figure 3).
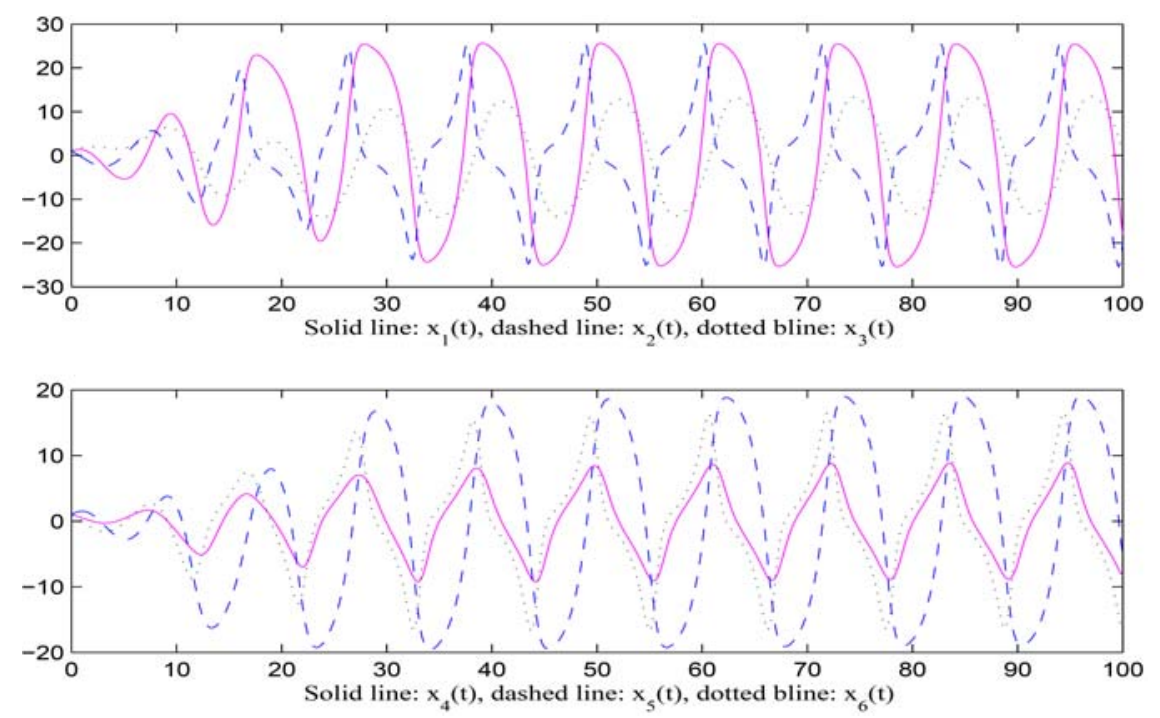

Figure 1. Dynamical behaviour of the solutions. delays: $(0.4,4,3)$. 

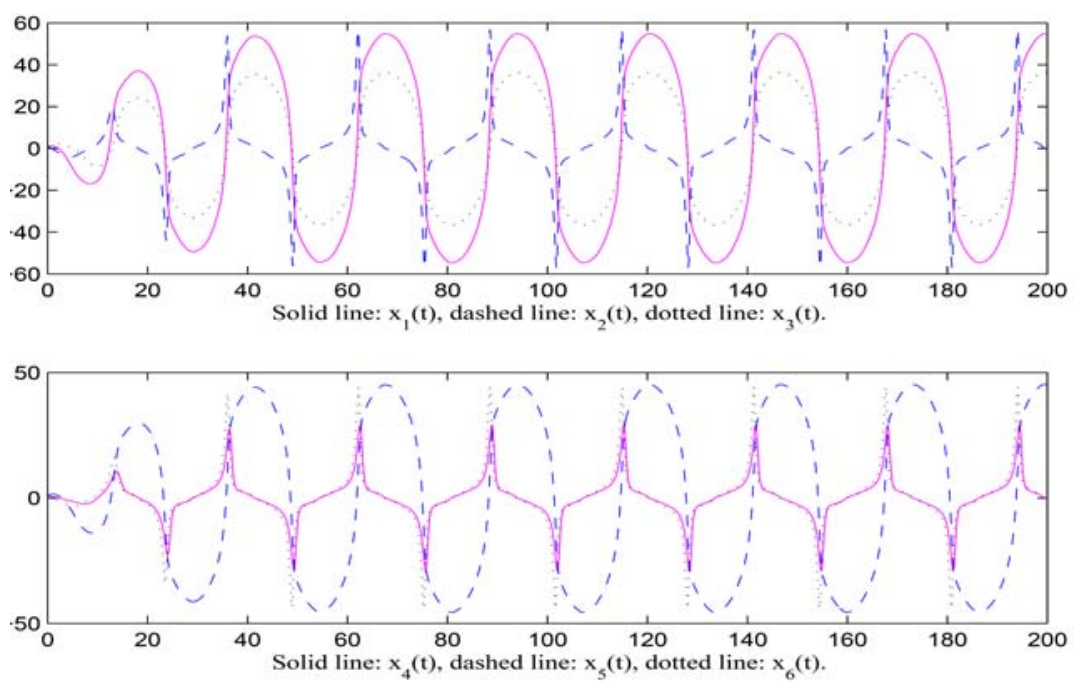

Figure 2. Dynamical behaviour of the solutions, delays: $(5,6,8)$.
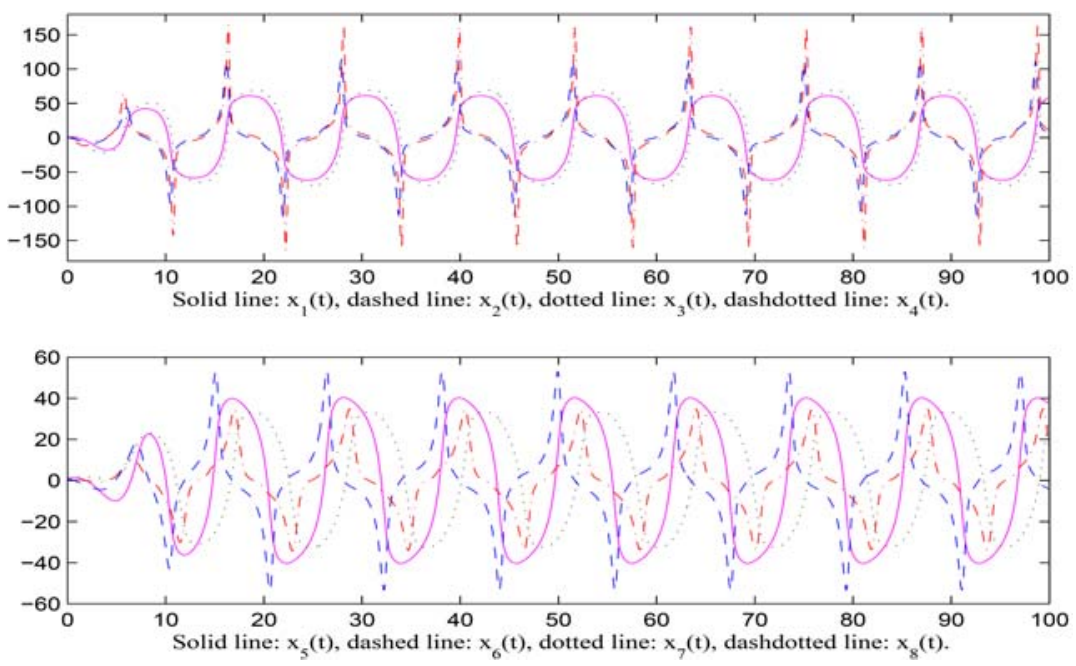

Figure 3. Dynamical behaviour of the solutions, delays: (1.5, 3.5, 1.5, 1.8). 


\section{Conclusion}

In this paper, we have discussed the dynamical behaviour of $n$ coupled van der Pol equation with time delays. Based on the mathematical analysis theory, a simple criterion to guarantee the existence of permanent oscillations, which is easy to check, as compared to the bifurcating method has been proposed. Some simulations are provided to indicate the correctness of the criterion.

\section{References}

[1] N. Hirano and S. Rybicki, Existence of limit cycles for coupled van der Pol equations, J. Diff. Equation 195(2) (2003), 194-209.

[2] R. H. Rand and P. J. Holmes, Bifurcations of periodic motions in two weakly coupled van der Pol oscillators, Internat. J. Nonlinear Mech. 15 (1980), 387-399.

[3] D. W. Stoliti and R. H. Rand, Dynamics of two strongly coupled relaxation oscillators, SIAM J. Math. Anal. 46(1) (1986), 56-67.

[4] A. Maccari, Response control for the externally excited van der Pol oscillator with non-local feedback, J. Sound and Vibration 331(5) (2012), 987-995.

[5] Z. M. Ge, S. C. Li, S. Y. Li and C. M. Chang, Pragmatical adaptive chaos control from a new double van der Pol system to a new double Duffing system, Appl. Math. Comput. 203(2) (2008), 513-522.

[6] Y. H. Qian and S. M. Chen, Accurate approximate analytical solutions for multidegree-of-freedom coupled van der Pol-Duffing oscillators by homotopy analysis method, Commun. Nonlinear Sci. Numer. Simul. 15(10) (2010), 3113-3130.

[7] S. Das and K. Maharatna, Fractional dynamical model for the generation of ECG like signals from filtered coupled van-der Pol oscillators, Computer Methods Programs in Biomedicine 112(3) (2013), 490-507.

[8] K. Rompala, R. Rand and H. Howland, Dynamics of three coupled van der Pol oscillators with application to circadian rhythms, Commun. Nonlinear Sci. Numer. Simul. 12(5) (2007), 794-803.

[9] H. G. Kadji, J. B. Orou and P. Woafo, Synchronization dynamics in a ring of four mutually coupled biological systems, Commun. Nonlinear Sci. Numer. Simul. 13 (7) (2008), 1361-1372.

[10] S. Wirkus and R. Rand, The dynamics of two coupled van der Pol oscillators with delay coupling, Nonlinear Dyn. 30(2) (2002), 205-221.

[11] X. Li, J. Ji and C. H. Hansen, Dynamics of two delay coupled van der Pol oscillators, Mech. Res. Commun. 33(5) (2006), 614-627. 
[12] J. M. Zhang and X. S. Gu, Stability and bifurcation analysis in the delay-coupled van der Pol oscillators, Appl. Math. Model. 34(9) (2010), 2291-2299.

[13] W. Y. Wang and L. J. Chen, Weak and non-resonant double Hopf bifurcations in $m$ coupled van der Pol oscillators with delay coupling, Appl. Math. Model. 39(10-11) (2015), 3094-3102.

[14] A. M. Atay, Van der Pol's oscillator under delayed feedback, J. Sound Vib. 218(2) (1999), 333-339.

[15] X. Li, J. C. Ji, C. H. Hansen and C. X. Tan, The response of a Duffing-van der Pol oscillator under delayed feedback control, J. Sound and Vibration 291(3-5) (2006), 644-655.

[16] I. V. Ermakov, G. V. Sande and J. Danckaert, Semiconductor ring laser subject to delayed optical feedback: Bifurcations and stability, Commun. Nonlinear Sci. Numer. Simul. 17(12) (2012), 4767-4779.

[17] H. B. Jiang, Q. S. Bi and S. Zheng, Impulsive consensus in directed networks of identical nonlinear oscillators with switching topologies, Commun. Nonlinear Sci. Numer. Simul. 17(1) (2012), 378-387.

[18] Y. H. Qian and S. M. Chen, Accurate approximate analytical solutions for multidegree-of-freedom coupled van der Pol-Duffing oscillators by homotopy analysis method, Commun. Nonlinear Sci. Numer. Simul. 15(10) (2010), 3113-3130.

[19] Y. Q. Li, W. H. Jiang and H. B. Wang, Double Hopf bifurcation and quasi-periodic attractors in delay-coupled limit cycle oscillators, J. Math. Anal. Appl. 387(2) (2012), 1114-1126.

[20] I. D. Landau, F. Bouziani, R. R. Bitmead and A. V. Besançon, Analysis of control relevant coupled nonlinear oscillatory systems, European J. Control 14(4) (2008), 263-282.

[21] Z. L. Shen and C. R. Zhang, Double Hopf bifurcation of coupled dissipative Stuart Landau oscillators with delay, Appl. Math. Comput. 227(15) (2014), 553-566.

[22] J. Forde and P. Nelson, Applications of Sturm sequences to bifurcation analysis of delay differential equation models, Preprint 2004.

[23] D. Ghosh, A. R. Chowdhury and P. Saha, On the various kinds of synchronization in delayed Duffing-van der Pol system, Commun. Nonlinear Sci. Numer. Simul. 13(4) (2008), 790-803.

[24] N. Chafee, A bifurcation problem for a functional differential equation of finitely retarded type, J. Math. Anal. Appl. 35(2) (1971), 312-348. 